\title{
Geoarchaeology or the contribution of geosciences for studying past human societies
}

\author{
Julien Curie ${ }^{1,2, *}$, Ségolène Vandevelde ${ }^{3,4}$, Amélie Quiquerez ${ }^{5}$ and Christophe Petit ${ }^{3,4}$ \\ ${ }^{1}$ CNRS, UMR7041 ArScAn/MSH Mondes, 21, allée de l'Université, 92023 Nanterre Cedex, France \\ 2 UMR6298 ARTEHIS, CNRS/uB, 6 Bd Gabriel, 21000 Dijon, France \\ ${ }^{3}$ Université Paris 1-Panthéon-Sorbonne, UMR7041 ArScAn, équipe Archéologies environnementales, Paris, France \\ ${ }^{4}$ MSH Mondes, 21, allée de l’Université, 92023 Nanterre Cedex, France \\ ${ }^{5}$ Université de Bourgogne, UMR6298 ARTEHIS, CNRS/uB, 6 Bd Gabriel, 21000 Dijon, France
}

Received: 9 June 2021 / Accepted: 10 June 2021 / Publishing online: 3 August 2021

\section{Introduction to the Special Issue "Geosciences and Human-Environment interactions"}

This special issue of the BSGF-Earth Sciences Bulletin dedicated to "Geosciences and Human-Environment interactions" gathers ten research papers that come out of the Geoarchaeology \& Environment session of the 26th edition of "Réunion des Sciences de la Terre" (Earth Sciences Meeting, "Société Géologique de France"/French Geological Union, Lille, France, 22nd-26th October 2018). With more than thirty abstracts submitted on this topic and with its thirty accepted oral presentations, this session has highlighted the growing interest for Geoarchaeology.

In France, the last decades have seen the emergence and increase of trans-disciplinary collaborations between earth scientists and archaeologists, as well as the merge of these to disciplines into integrated geoarchaeological approach. Geoarchaeology uses multidisciplinary approaches developed in Earth Sciences to study the interrelations between human societies and their environment over the long term. Its main goal is to contribute to a better understanding of the spatial and temporal patterns of interactions and/or relationships between societies and environment.

Geoarchaeology - considered here as "Geosciences for studying Past Human Societies" - covers a wide range of scopes and encompasses multiple thematic areas such as: locating and detecting archaeological sites in their environmental and geomorphological contexts, analysing local and regional stratigraphic and microstratigraphic records, studying anthropogenic vs "natural" sediments and landforms, reconstructing site formation processes and the underlying human impact on environment, producing paleoenvironmental and chronological interpretations, studying artefacts and ecofacts for elucidating resource origin, manufacturing practices and cultural networks, etc.

This special issue explores all these research topics in ten papers grouped into three sets. (1) The first set of papers presents intra-site analyses carried out on the sedimentary archives of settlement sites in Palaeolithic and Medieval contexts (respectively Vandevelde et al. and Borderie et al.); (2) the second set presents provenance studies that aim to track the origin of various types of materials (metal, stones, ceramic) from Neolithic, Antique and Pre-Columbian archaeological sites (respectively Tomczyk et al.; Vals et al.; Fronteau and van den Bel); (3) and the third set presents analyses of the evolutions of such environments and landscapes (Schaal and Naton; Ollive et al.; Gouriveau et al.; Deschodt et al.; Blond et al.).

\section{What analyses carried out on sedimentary archives of settlement sites can tell us about human activities?}

The paper from Vandevelde et al. presents a geoarchaeological study of finely laminated parietal carbonated crusts of the Grotte Mandrin Palaeolithic site (southern France). Using LIBS micro-spectroscopy, the authors propose to reconstruct palaeo-fire chronicles and to set them up on a micro-chronological timescale (with annual resolution), thus developing a new research area: the fuliginochronology. This method and the resolution they reach allow the authors to access new information like the precise timing of occupations on the site.

\footnotetext{
*Corresponding author: julien.curie@sorbonne-universite.fr
} 
The next paper focuses on much more recent and urbanised context. Observed in several urban archaeological stratifications, the "Dark Earth" formations are major common medieval pedo-sediments seen as archives of past human activities in inhabited areas. By taking them into account in several archaeological stratifications from Medieval urban sites all over Europe and by using a multidisciplinary approach (underlying the organic carbon, phosphorous and heavy metals contents of these sedimentary deposits), the paper from Borderie et al. improves our acknowledgment of early medieval urban societies, teaching about their way of lives, especially their waste management.

\section{What can material analyses can tell us about provenance?}

Through a case study on Neolithic copper mines, the paper from Tomczyk et al. presents multivariable statistical methods applied on lead isotopes coming from gitological data and geoarchaeological database in order to improve the tracing of copper artefacts' production sources.

In the second paper about provenance studies, Vals et al. present an exhaustive study of the origins of the stones used in the famous Sanctuary of Apollo in Delphi, Greece (known for its Oracle during Greek Antiquity). They provide an identification of the stone facies employed in the archaeological site as well as of their origin.

The petrographical analysis performed on ceramics from three pre-Columbian sites on the archipelago of Guadeloupe (French West Indies) by Fronteau and van den Bel demonstrates the presence of grog used as temper and convincingly conclude about the origin and the spreading of this true regional chrono-cultural marker.

\section{How to reconstruct the evolution of environments?}

The archaeobotanical study performed by Schaal and Naton on the sediments that accumulated in an oxbow-lake of the River "Meuse" (northeast of France) led to build a true and strong taphonomic reference frame that constrains taphonomic processes and criteria preservation of the ecofacts and improves our knowledge of the palaeoecology of the Preboreal period (11.7-10.7 ka BP).

The paper from Ollive et al. provides new features on the so-long debate about the closed depression (named "mardelles") observed on Keuper marls of the northeastern Paris Basin. Thanks to rescue archaeological excavations along the high-speed train line "LGV-Est Européenne" together with a multidisciplinary approach, the authors demonstrate the natural origin of these structures and precisely describe the geological processes involved, including the human-induced sedimentary filling.

The paper from Gouriveau et al. focuses on reconstructing palaeo-environments and human-environment interactions throughout the Holocene (since $6600 \mathrm{cal}$. BP to Middle-Age) in the northeast of France from a multidisciplinary palaeoenvironmental study performed in bog-pond located near the ruins of the medieval castle of Waldeck (Northern Vosges, France).

Following the recent expansion project of the Dunkerque's harbor (north of France), Deschodt et al. investigated the past estuary of a sub-basin of the coastal River "Aa" thanks to rescue archaeological operations. Through the use of trans-disciplinary methods (historical archives, lithostratigraphic analysis, electrical conductivity mapping), the authors propose a reconstitution of the landscape evolution and the human occupations in the area since the 10th century CE.

Blond et al. propose a reconstitution of environmental and landscape changes around the archaeological site of Wakarida (Ethiopia). From an approach combining field measurements, laboratory analyses, dating performed on colluvial and alluvial, they discuss how climate and anthropogenic processes have both contributed to reshape the landscapes through Early and Middle Holocene and during the Classical Aksumite (150-400/450 CE) and the post Aksumite (800/850 CE) periods.

This special issue brings together a truly diverse group of scholars showing their efforts in developing, testing, and debating methodologies in the domain of Geoarchaeology. The geoarchaeological approach must work on breaking down the barriers between disciplinary approaches and foster integrative approaches leading to the production, in many ways, of scientific features focusing on Societies-Environments interactions.

Acknowledgments. We would like to acknowledge the editorial board of the BSGF-Earth Sciences Bulletin, Pr. Laurent Jolivet (Sorbonne University), Editor-in-Chief, and Solen Le Gardien (SGF) from the Editorial Office. We want to thank all the authors for their contributions to this volume. We would like to gratefully thank the referees involved in the review process and who significantly contributed to the quality of this special issue.

\section{La géoarchéologie ou l'apport des géosciences pour l'étude des sociétés humaines du passé}

\section{Introduction au numéro spécial «Géosciences et interactions humain-environnement »}

Ce numéro spécial du $B S G F$-Earth Sciences Bulletin consacré aux "Géosciences et interactions humain-environnement » rassemble dix articles de recherche issus de la session Géoarchéologie \& Environnement de la $26^{\mathrm{e}}$ édition de la Réunion des Sciences de la Terre (Société Géologique de France, Lille, France, 22-26 octobre 2018). Avec plus d'une trentaine de présentations orales acceptées, cette session a mis en évidence l'intérêt croissant des Sciences de la Terre pour la Géoarchéologie. 
En France, les dernières décennies ont vu l'émergence et l'augmentation des collaborations transdisciplinaires entre les géoscientifiques et les archéologues, ainsi que la fusion de ces deux disciplines dans une approche géoarchéologique intégrée. Cette dernière utilise des approches multidisciplinaires développées dans les sciences de la Terre pour étudier les relations entre les sociétés humaines et leur environnement sur le temp long. Son objectif principal est de contribuer à une meilleure compréhension des schémas spatiaux et temporels des interactions entre les sociétés et l'environnement.

La géoarchéologie - considérée ici en tant que «géosciences pour l'étude des sociétés humaines du passé »-couvre un large éventail de champs d'application et englobe de multiples domaines thématiques tels que : la localisation et la détection des sites archéologiques dans leurs contextes environnementaux et géomorphologiques, l'analyse des enregistrements stratigraphiques et microstratigraphiques locaux et régionaux, l'étude des sédiments anthropiques $v s$ naturels et des formes de paysages, la reconstitution des processus de formation des sites et de l'impact humain sur l'environnement, la production d'interprétations paléoenvironnementales et chronologiques, l'étude des artefacts et des écofacts pour élucider l'origine des ressources, les pratiques de fabrication et les réseaux culturels, etc.

Ce numéro thématique explore tous ces sujets de recherche dans dix articles regroupés en trois ensembles. (1) La première série présente des analyses intra-sites effectuées sur les archives sédimentaires de sites occupés dans des contextes paléolithiques et médiévaux (respectivement Vandevelde et al. et Borderie et al.); (2) la deuxième série présente des études de provenance qui visent à retracer l'origine de divers types de matériaux (métal, pierres, céramique) provenant de sites archéologiques néolithiques, antiques et précolombiens (respectivement Tomczyk et al.; Vals et al.; Fronteau et van den Bel); (3) et le troisième ensemble présente des analyses des évolutions d'environnements et de paysages (Schaal et Naton; Ollive et al. ; Gouriveau et al. ; Deschodt et al.; Blond et al.).

\section{Que peut nous apprendre l'analyse d'archives sédimentaires de sites de peuplement sur les activités humaines?}

L'article de Vandevelde et al. présente une étude géoarchéologique d'encroûtements carbonatés pariétaux finement laminés provenant du site paléolithique de la Grotte Mandrin (sud de la France). En utilisant la micro-spectroscopie LIBS, les auteurs proposent de reconstruire des chroniques des paléo-feux et de les caler sur une échelle de temps micro-chronologique (avec une résolution annuelle), développant ainsi un nouveau domaine de recherche : la fuliginochronologie. Cette méthode et la résolution qu'elle atteint permettent aux auteurs d'accéder à de nouvelles informations comme la fréquence précise des occupations du site.

L'article suivant se concentre sur un contexte beaucoup plus récent et urbanisé. Observées dans plusieurs stratigraphies archéologiques urbaines, les formations de «Terres noires» sont des pédo-sédiments médiévaux majeurs considérés communément comme des archives des activités humaines passées. À partir de différents exemples de stratigraphies archéologiques de sites médiévaux à travers l'Europe et en utilisant une approche multidisciplinaire (basée sur les contenus en carbone organique, phosphore et métaux lourds de ces dépôts sédimentaires), l'article de Borderie et al. aide à mieux comprendre les sociétés urbaines du haut Moyen-Âge, en renseignant leur mode de vie et en particulier la gestion des déchets.

\section{Que peut nous apprendre l'analyse des matériaux sur leur provenance?}

À travers une étude de cas sur les mines de cuivre néolithiques, l'article de Tomczyk et al. présente des méthodes statistiques multivariées appliquées aux isotopes du plomb provenant de données gîtologiques et de bases de données géoarchéologiques afin d'améliorer le traçage des sources de production des artefacts en cuivre.

Dans le second article sur les études de provenance, Vals et al. présentent une étude exhaustive de l'origine des pierres utilisées dans le célèbre sanctuaire d'Apollon à Delphes, en Grèce (connu pour son Oracle durant l'Antiquité grecque). Les auteurs fournissent une identification des faciès des pierres utilisées dans le site archéologique ainsi que de leur origine.

L'analyse pétrographique réalisée sur des céramiques provenant de trois sites précolombiens de l'archipel de la Guadeloupe (Antilles françaises) par Fronteau et van den Bel démontre la présence de chamotte utilisée comme dégraissant et conclut, de façon convaincante, sur l'origine et la diffusion de ce véritable marqueur chrono-culturel régional.

\section{Comment reconstituer l'évolution des environnements?}

L'étude archéobotanique réalisée par Schaal et Naton sur les sédiments accumulés dans un bras mort de la Meuse (nord-est de la France) a permis de construire un solide cadre de référence taphonomique de préservation des écofacts et améliore notre connaissance de la paléoécologie de la période préboréale (11.7-10.7 ka BP).

L'article d'Ollive et al. apporte de nouveaux éléments au long débat sur les dépressions fermées (appelées «mardelles») observées dans les marnes de Keuper du nord-est du Bassin parisien. Grâce à des opérations archéologiques préventives le long de la ligne «LGV-Est européenne » et à une approche pluridisciplinaire, les auteurs démontrent l'origine naturelle de ces structures et décrivent précisément les processus géologiques impliqués, y compris le remplissage sédimentaire induit par les activités humaines.

L'article de Gouriveau et al. se concentre sur la reconstitution des paléo-environnements et des interactions hommeenvironnement tout au long de l'Holocène (depuis $6600 \mathrm{cal}$. BP jusqu'au Moyen-Âge) dans le nord-est de la France à travers une 
étude paléo-environnementale multidisciplinaire réalisée dans un étang tourbeux situé près des ruines du château médiéval de Waldeck (Vosges du Nord, France).

Suite au récent projet d'expansion du port de Dunkerque (nord de la France), Deschodt et al. ont étudié l'ancien estuaire d'un sous-bassin de la rivière côtière «Aa» grâce à des opérations archéologiques préventives. Grâce à l'utilisation de méthodes transdisciplinaires (archives historiques, analyse lithostratigraphique, cartographie de la conductivité électrique), les auteurs proposent une reconstitution de l'évolution du paysage et des occupations humaines dans la région à partir du $\mathrm{X}^{\mathrm{e}}$ siècle de notre ère.

Blond et al. proposent une reconstitution des changements environnementaux et paysagers autour du site archéologique de Wakarida (Éthiopie). À partir d'une approche combinant des mesures de terrain, des analyses de laboratoire, des datations réalisées sur des colluvions et des alluvions, les auteurs discutent de la manière dont les processus climatiques et anthropiques ont contribué à modeler les paysages à l'Holocène ancien et moyen, et durant les périodes axoumite (150-400/450 AD) et postaxoumite $(800 / 850 \mathrm{AD})$.

Ce numéro spécial réunit un panel de scientifiques très diversifiés qui montrent leurs efforts pour développer, tester et débattre des méthodologies dans le domaine de la géoarchéologie. L'approche géoarchéologique doit s'efforcer de construire et renforcer les liens entre les approches disciplinaires et doit s'attacher à favoriser les approches intégratives multiples, susceptibles de produire des réflexions, des travaux et des résultats scientifiques autour des interactions Sociétés-Environnements.

Remerciements. Nous tenons à remercier le comité de rédaction du BSGF-Earth Sciences Bulletin, Pr. Laurent Jolivet (Sorbonne Université), rédacteur en chef, et Solen Le Gardien (SGF) du bureau de rédaction. Nous tenons à remercier tous les auteurs pour leur contribution à ce volume. Nous tenons également à remercier chaleureusement les reviewers qui ont participé au processus de révision des articles et qui ont contribué de manière significative à la qualité de ce numéro spécial. 